Paediatr Paedolog 2017 · 52 (Suppl 1):S1-S3 DOI 10.1007/s00608-017-0483-z

Online publiziert: 19. Juni 2017

๑) Springer-Verlag Wien 2017

CrossMark

\author{
Johann Deutsch' • Reinhold Kerbl' . Christian Popow ${ }^{3}$ Ernst Tatzer ${ }^{4}$. \\ Leonhard Thun-Hohenstein ${ }^{5}$ \\ 1 Politische Kindermedizin, Graz, Österreich \\ ${ }^{2}$ Abteilung für Kinder und Jugendliche, LKH Hochsteiermark/Leoben, Leoben, Österreich \\ ${ }^{3}$ Universitätsklinik für Kinder- und Jugendpsychiatrie, Medizinische Universität Wien, Wien, Österreich \\ ${ }^{4}$ NÖGUS, NÖ Kinder- und Jugendplan, Hinterbrühl, Österreich \\ ${ }^{5}$ Universitätsklinik für Kinder- und Jugendpsychiatrie, Salzburger Landeskliniken/Paracelsus Medizinische \\ Privatuniversität, Christian Dopplerklinik, Salzburg, Österreich
}

\title{
Im Netzwerk geborgen? Netzwerke und ihre Wirkung
}

\author{
10. Jahrestagung der Politischen \\ Kindermedizin, 11.-12.11.2016
}

Die Kooperation zwischen den an der Behandlung von erkrankten Kindern und Jugendlichen Beteiligten hängt derzeit meist noch immer vom persönlichen Engagement Einzelner ab. Um ein höheres Niveau in der Betreuung kranker Kinder und Jugendlicher zu erreichen, ist neben einer stärkeren Spezialisierung gleichzeitig eine systematische starke Vernetzung mit Kollegen der verschiedenen Fachbereiche und allen Personen/ Berufen notwendig, die für das Wohlergehen der Kinder und Jugendlichen und für ihre Zukunftsaussichten eine entscheidende Rolle spielen. Ein Bereich, in dem diese Vernetzung von allem Anfang schon ersichtlich war, ist die Kinder- und Jugendpsychiatrie/ Heilpädagogik/Sozialmedizin. Bis zu dem Zeitpunkt, wo die Jugendlichen die Verantwortung für die Behandlung ihrer (meist chronischen) Erkrankung selbst übernehmen können, ist die intensive Einbindung der Eltern bzw. der für die Kinder Verantwortlichen entscheidend für deren Zukunft. Dies bedeutet derzeit aber noch, dass die betroffenen Familien sich mit einer Vielzahl von Hilfen und Helfern konfrontiert sehen, die manchmal noch so agieren, als wären sie alleinige Betreuer dieser Familie. Für die Transition der Jugendlichen in die Betreuung von Erwachsenenmedizinern ist eine gut funktionierende Vernetzung besonders entscheidend, wie bereits im letzten Supplement zur Jahrestagung 2015 der Politischen Kindermedizin (PKM; [1]) dargestellt wurde. Vernetztes Arbeiten und systematische intensive Kooperation sind auch nötig, um Parallelen, Wiederholungen und gelegentlich auch Blockaden zu vermeiden.

\section{》) Vernetzung bezieht sich auch auf Wissenschaft und Planung von Versorgungssystemen}

Die heutige Kindermedizin versucht mehr denn je eine „person-centered care" anzubieten, d. h. das Kind und sein Umfeld ins Zentrum der Betreuung zu stellen und so nicht nur die Krankheit zu behandeln, sondern die Möglichkeit $\mathrm{zu}$ bieten, als Individuum ein integriertes Leben mit einer Erkrankung leben zu können. Vernetzung bezieht sich neben der direkten Versorgung der Menschen auch auf die Wissenschaft und die Planung von Versorgungssystemen. Im Rahmen der 10. Jahrestagung der PKM haben wir versucht, diesen Bogen bis zur politischen Wirkung einer Vernetzung der Zivilgesellschaft zum Wohl der Kinder und Jugendlichen zu spannen. Im Beitrag des ehemaligen Wissenschaftsministers werden wir zuletzt aufgefordert, durch die gute Vernetzung mit Fachleuten und Politik das in unseren Reihen vorhandene Fachwissen nicht nur für die Gesundheit der Kinder und Jugendlichen, sondern uns auch für eine Änderung der häufig negativen Einstellung der Gesellschaft gegenüber Kindern und Jugendlichen einzusetzen und uns dabei durch eventuelle Rückschläge nie entmutigen $\mathrm{zu}$ lassen. Denn von eben dieser Einstellung der Gesellschaft hängt auch deren Zukunft direkt ab! Die ebenfalls am ersten Tag der Tagung anwesende Abgeordnete des Nationalrats und ehemalige Frauen- und Bildungsministerin will diese unsere Anliegen ebenfalls intensiv unterstützen.

Fast alle Vortragenden der 10. PKMJahrestagung haben ihre vor Ort getroffenen Aussagen in den folgenden Manuskripten zusammengefasst, um damit eine ausreichende Breitenwirkung und stärkere systematische Vernetzung zum Wohl der von uns betreuten Kinder und Jugendlichen $\mathrm{zu}$ erzielen.

Wir danken allen Beitragenden für deren Engagement im Sinn der Kinder und Jugendlichen und dem SpringerVerlag für die neuerlich gute Kooperation bei der Erstellung dieses Supplements.

Unseren geneigten Leserinnen und Lesern wünschen wir viel Freude bei der Lektüre der Beiträge. Wir freuen uns über jede Form der konstruktiven 


\section{Editorial}

Rückmeldung, gegebenenfalls aber auch auf eine aktive Beteiligung in der Politischen Kindermedizin.

Die Herausgeber

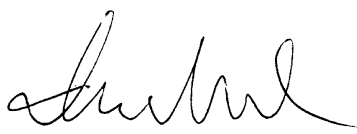

J. Deutsch

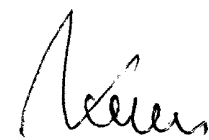

R. Kerbl

\section{Cenistion Porow}

C. Popow

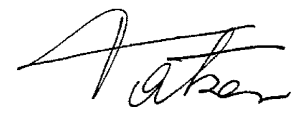

E. Tatzer

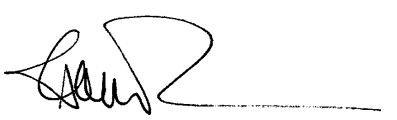

L. Thun-Hohenstein

\section{Korrespondenzadresse}

Univ.-Prof. Dr. J. Deutsch
Politische Kindermedizin
Schaftalstraße 41, $8010 \mathrm{Graz}$,
Österreich
jeejrm@posteo.de

Interessenkonflikt. J. Deutsch, R. Kerbl, C. Popow,

E. Tatzer und $L$. Thun-Hohenstein geben an, dass kein Interessenkonflikt besteht.

\section{Literatur}

1. Deutsch J, Gobara S, Waldhauser F (2016) Lost in Transition - wenn aus Kindern Erwachsene werden. Pädiatr Pädol 51(Suppl. 1):S1-S75 
Hier steht eine Anzeige.

\section{曾 Springer}

